\title{
Secondary Stress based-Remaining Fatigue Life Estimation of a Riveted Bridge Connection using New Fatigue Criterion
}

\author{
$\bar{P}, \bar{B} . \bar{R} \cdot \bar{D}$ issanayake and $\bar{S} . \bar{C}$. Siriwardane
}

\begin{abstract}
This paper presents a new method to estimate the remaining fatigue lives of riveted connections of railway bridges. The proposed method mainly consists of secondary stress histories, recently developed sequential law and fully known Wöhler curve. It is essential to use the fully known Wohler curve. Therefore the technique, which utilizes the transfer of the partially known code given Wöler curve to a fully known curve, is also discussed. Initially the details of new damage indicator based sequential law and it's proposed extension for multiaxial fatigue is described. Secondly the remaining fatigue life of an existing riveted bridge connection is estimated by introducing a new method. Hence validity and merits of the proposed method is confirmed by comparing the results with previous methods-based fatigue lives.
\end{abstract}

Keywords: Riveted connection, Fatigue life, Sequential law

\section{Introduction}

At present, rail authorities all over the world are paying special attention to evaluate the remaining fatigue life of riveted railway bridges, siñce môșt of these bridges are nearing the end of their design fatigue lives [1]. Most of the present day fatigue assessment approaches of railway bridges are generally based on combination of primary stress histories under actual traffic load [2,3], Miner's rule [4] and railway code provided design fatigue curve (also referred to as S-N or Wöhler curve).

The primary stresses are usually determined by the global analysis or experimental measurement of real responses of the whole structure. In such situations, it cannot easily predict secondary stresses (stress concentration effects) around the discontinuities such as connections, notches or cracks especially in old bridges. Therefore in the present approach, S-N curves, which correspond to the detail class [5] of riveted connection, are considered to capture the fatigue damage due to the secondary stresses near the riveted connection or discontinuities. Even though, this detail class is determined by considering the quality of the workmanship, sometime it might give over or under prediction to real secondary stress state. As a result, fatigue lives estimated using the code given design S-N curves exhibits pessimistic results.
However, secondary stress effect in riveted connection between the primary members of bridges was found to to be ône ôf main reasons for fatiguie dàmáge $[6]$. Further it has been identified that the rotational fixity of riveted connection and the variation in the clamping force of rivets [7] are the major causes leading to fatigue cracking in riveted connection [1]. Therefore it is important to investigate accurately the fatigue damage due to secondary stresses at the riveted connections of existing

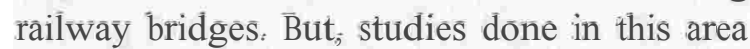
are few and most of the approaches were based on combination of secondary stress histories, equivalent effective stress with Miner's rule [4] and railway code provided mean fatigue curve. The Miner's rule has always been acknowledged as a simplification that is easy to use in design where detailed loading history is unknown. But under many variable amplitude loading conditions, life predictions have been found to be unreliable since it did not properly take into âccount the loading sequence effect [810]. Therefore, it is uncertain to use the Miner's rule for remaining fatigue life estimation of railway bridges because most of the railway bridges are subjected to variable amplitude loadings. Recently, a new damage indicatorbased sequential law [10] was developed to

Ettg (Dr) P.B.R. Dissanayake, C Eng; MIE(SL), B.Sc. Eng. (Hons) "(Perndeniya); MSć, (Eluine), PhD. (Ehime), Senior Lecture); Department of Civil Engineering, University ef Peradeniya. Pemdeniya. Sn'Lanka. S. C. Siriwardatte, B.Sc. Eng, (Hons) (Peradeniya), Mp 71 I (Peradeniya). 
capture the loading sequence effect of variable amplitude loads more precisely.

Therefore, the main objective of this paper is to estimate remaining fatigue life of a riveted railway bridge connection due to the effect of secondary stresses using the multiaxial sequential law. In this approach, fully known S$\mathrm{N}$ curve is essential to use as the related fatigue curve. The corresponding code provided mean curves, which were relevant to previous approaches, are partially known S-N curves. Therefore the technique, which utilizes transfer of the partially known Wöhler curve to a fully known curve, is discussed in this paper. Finally, the paper presents comparisons of obtained fatigue lives with the previous Miner's rulebased estimations.

\section{A New Damage Indicator based - Sequential Law}

A new damage indicator based sequential law [10] is used to obtain a more realistic fatigue life for bridges. The hypothesis behind this fatigue law is that if the physical state of damage is the same, then fatigue life depends only on loading condition. In the case of bridges, it can be said that if the connection detail type and environment are the same, only the loading condition matters in fatigue.

\subsection{Description of the Model}

A detailed description of the damage stress model and the definition of damage indicator, $D_{i}$ is described in the corresponding paper [10]. Here only the concept is summarized with an algorithm for comprehension (see flow chart described in Figure 1). Suppose a part is subjected to a certain stress amplitude or stress range $\sigma_{i}$ for $n_{i}$ number of cycles at load level $i$ and $N_{i}$ is the fatigue life (failure number of cycles) corresponding to $\sigma_{i}$ (see Figure 2). The residual life at load level $i$ can be obtained as $\left(N_{i}-n_{i}\right)$. The stress $\sigma_{(i) e q}$ which corresponds to the failure life $\left(N_{i}-n_{i}\right)$ is named as $i^{\text {th }}$ level damage stress amplitude or stress range (otherwise can be introduced as stress amplitude or stress range relevant to the residual life). Hence, the new damage indicator, Di is stated as,

$D_{i}=\frac{\sigma_{(i) e q}-\sigma_{i}}{\sigma_{u}-\sigma_{i}}$
Where $\sigma_{u}$ is the intercept of the Wöhler curve with the ordinate at one-quarter of first fatigue cycle. Furthermore, it can be stated that, $\sigma_{u}$ is the ultimate tensile strength amplitude or range for rotating bend test-based $S$ - $N$ curves and it is the ultimate shear strength amplitude or range for torsional fatigue test-based $S$ - $N$ curves.

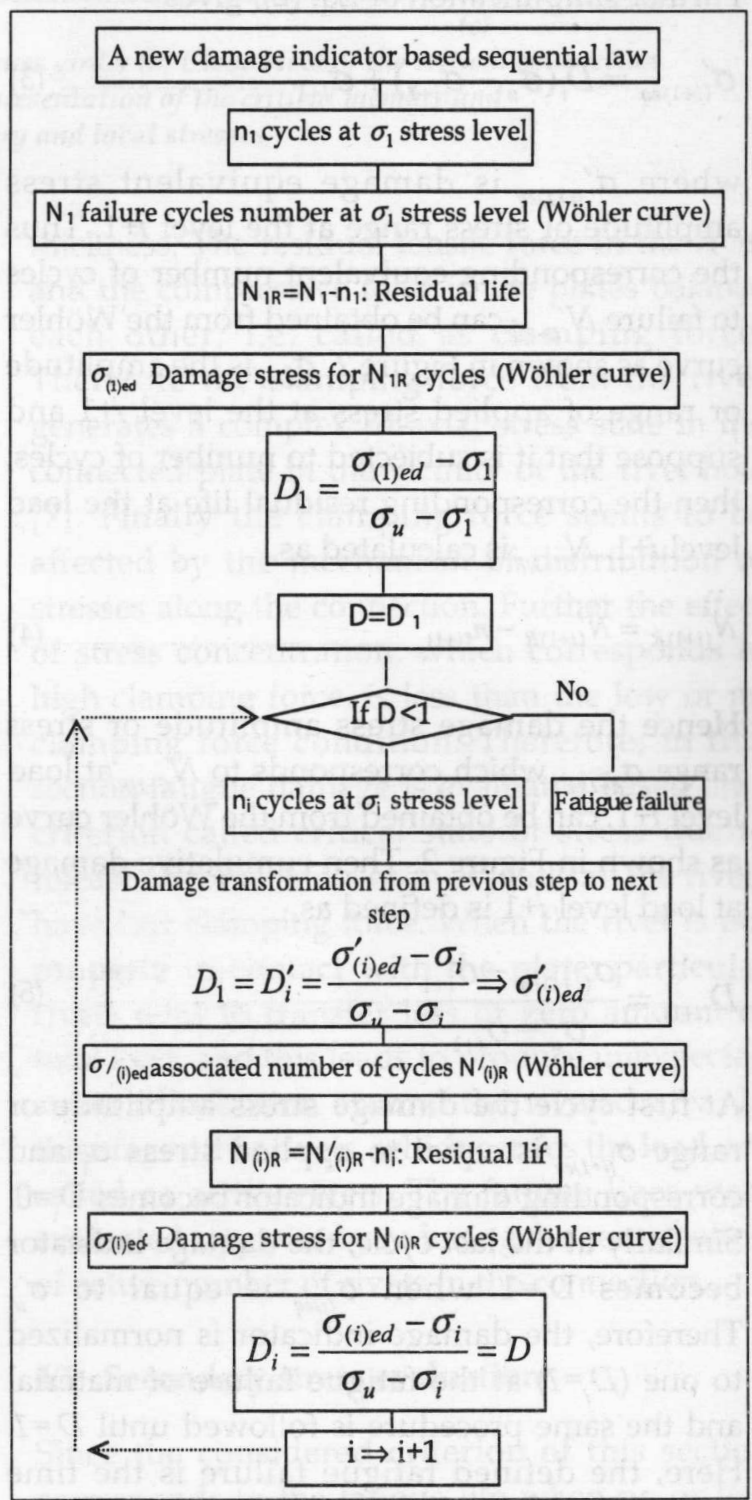

Figure 1 - Flow chart for damage stress based sequential law

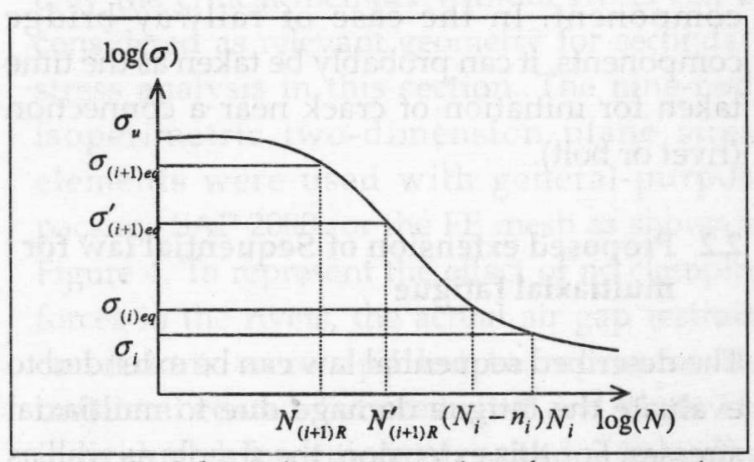

Figure 2 - Schematic representation of parameters in Wöhler curve 
The same damage is then transformed to load level $i+1$ and hence damage equivalent stress at level $i+1$ is calculated using the relation,

$D_{i}=\frac{\sigma_{(i) e q}-\sigma_{i}}{\sigma_{u}-\sigma_{i}}=\frac{\sigma_{(i+1) e q}^{\prime}-\sigma_{i+1}}{\sigma_{u}-\sigma_{i+1}}$

Further simplification of Eq. (2), gives

$\sigma_{(i+1) e q}^{\prime}=D_{i}\left(\sigma_{u}-\sigma_{i+1}\right)+\sigma_{i+1}$

where $\sigma_{(i+1) e q}^{\prime}$ is damage equivalent stress amplitude or stress range at the level $i+1$. Thus the corresponding equivalent number of cycles to failure $N_{(i+1) R}^{\prime}$ can be obtained from the Wöhler curve as shown in Figure 2. $\sigma_{u i+1}$ is the amplitude or range of applied stress at the level $i+1$ and suppose that it is subjected to number of cycles, then the corresponding residual life at the load level $i+1, N_{(i+1) R}$ is calculated as,

$N_{(i+1) R}=N_{(i+1) R}^{\prime}-n_{(i+1)}$

Hence the damage stress amplitude or stress range $\sigma_{(i+1) e q^{\prime}}$ which corresponds to $N_{(i+1) R}$ at load level $i+1$, can be obtained from the Wöhler curve as shown in Figure 2. Then cumulative damage at load level $i+1$ is defined as,

$D_{(i+1)}=\frac{\sigma_{(i+1) e q}-\sigma_{i+1}}{\sigma_{u}-\sigma_{i+1}}$

At first cycle the damage stress amplitude or range $\sigma_{(i+1) e q}$ is equal to applied stress $\sigma_{1}$ and corresponding damage indicator becomes $\mathrm{D}_{i}=0$. Similarly at the last cycle, the damage indicator becomes $\mathrm{D}_{i}=1$ when $\sigma_{(i) e q}$ is equal to $\sigma_{u}$. Therefore, the damage indicator is normalized to one $\left(D_{i}=1\right)$ at the fatigue failure of material and the same procedure is followed until $D_{i}=1$. Here, the defined fatigue failure is the time taken to first through-thickness crack at the location of maximum stress of the structural component. In the case of railway bridge components, it can probably be taken as the time taken for initiation of crack near a connection (rivet or bolt).

\subsection{Proposed extension of Sequential law for multiaxial fatigue}

The described sequential law can be extended to evaluate the fatigue damage due to multiaxial stresses. For this extension, the simple as well as the commonly used approach has been considered in our study as directed in the corresponding paper [10]. It basically consists of transforming cyclic multiaxial stresses into equivalent uniaxial stress (generally called as effective stress which characterizes the deformation of the material) amplitude or range, which should generate the same fatigue life as that due to the multiaxial stresses. The equivalent stress amplitude or range is then used to enter a uniaxial $S-N$ curve to determine the damage indicator $D_{i}$ of the sequential law. The usual methods for making such transformations are extensions of Von Mises (octahedral shear stress) or Tresca (maximum shear stress) yield criteria for proportional loading conditions [8,10-12]. Here, Von Mises yield criteria is used for the transformation and multiaxial stress fields at critical locations were considered in terms of equivalent von Misses stress amplitudes or ranges. The steps followed are briefly summarized as follows.

- First select the mean $S-N$ curve, which represents no effect of the clamping force, from the UK railway assessment code [13].

- The von Misses stress (effective stress) histories due to passage of trains for critical location are obtained.

- Then the von Mises stress histories are converted into effective stress ranges and effective mean stress by using the reservoir counting method [5].

- The modified Goodman equation [8] was used to do the mean stress correction of each effective stress range. The effective stress ranges were then transferred to equivalent fully reversed uniaxial cyclic stress ranges.

- Finally the damage indicator Di was obtained and the remaining fatigue life was estimated for critical location by following the procedure mentioned in sub section 2.1.

Here it is important to note that the described procedure is applicable to critical points, which are subjected to proportional loading conditions. In other words it can be stated that this procedure is applicable when the state of stress is at the elastic limit for tension fatigue. 


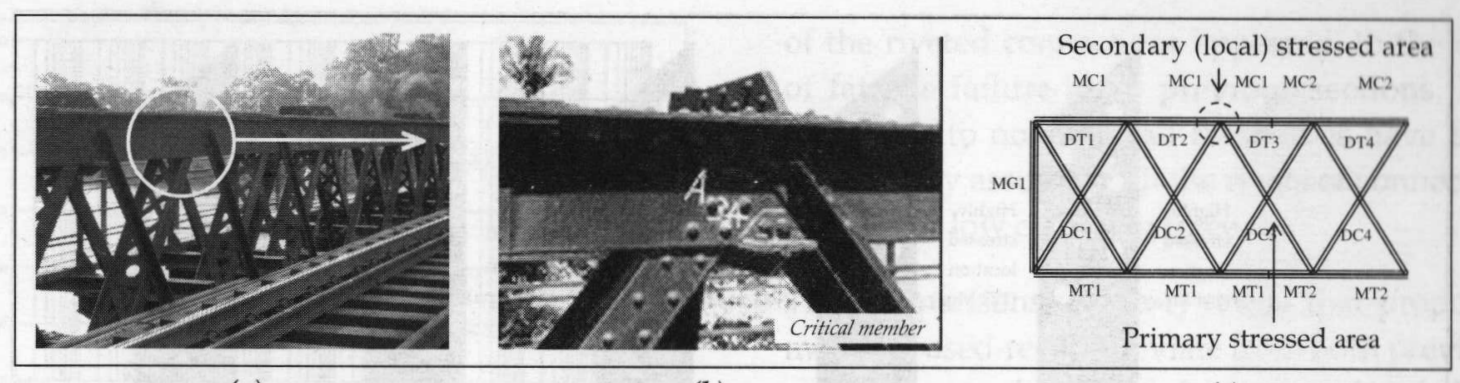

(a)

(b)

(c)

Figure 3-(a) Critical riveted connection of the main truss girder (b) Close view of the critical connection and the critical member (c) Schematic representation of the critical member and related areas for primary and local stresses

\section{Considered Riveted Connection of a Railway Bridge}

The considered riveted connection was selected corresponding to a highly stressed member, which was found from the detailed investigation of one of the longest railway bridges in Sri Lanka [14]. Since this connection shows the lowest remaining fatigue life of the main truss girder, it was introduced as critical connection of the truss. The selected connection with highly stressed member is shown in Figure 3. The condition survey [14] reveals that there are no visual cracks in any component of the connection. In-situ measurements of member sizes and connections verified the fact that the existing drawings were applicable. Further it can be said that comparatively the maintenance work carried out on this connection thus far is satisfactory.

\section{Remaining Fatigue Life Evaluation}

Remaining fatigue life evaluation of a considered riveted connection is discussed in this section and evaluations are especially based on secondary stresses, which are generated around the riveted connection due to stress concentration effect of primary stress. The considered secondary stresses are usually subjected to multiaxial state of stress. To evaluate the so-called secondary stresses, the riveted area of the member has to be subjected to further fine mesh FEM analysis.

When a hot rivet is inserted into the hole of plates in order to connect them and when the second head is formed from the protruding shank, the rivet gets shortened in length due to cooling. However most of shrinkage of the free rivets is restricted by the connected plates, which consequently are compressed through the thickness. The residual tensile force in the rivet and the compressive force in the plates balance each other; i.e. called as clamping force. Therefore the clamping force from the rivet generates a complex tri-axial stress state in the connected plate in the vicinity of the rivet hole [7]. Finally the clamping force seems to be affected by the mechanism of distribution of stresses along the connection. Further the effect of stress concentration, which corresponds to high clamping force, is less than the low or no clamping force condition. Therefore, in this section fatigue damage is evaluated based on a criterion called critical state of stress due to release of contactness of rivet while all the rivets have low clamping force. When the rivet is not properly in contact with the plate, particular rivets tend to transfer less or zero amount of total load, and this leads to produce unexpected stress distribution around the riveted area. In this stage other rivets, which carries the load, are called as active rivets. The fatigue lives were evaluated stepwise by reducing the contribution of active number of rivets in the connection.

\subsection{Secondary stress evaluation}

Since the considered criterion of this section corresponds to the fatigue life when no or low clamping force at the rivets as mentioned before, only the critical member without rivets can be considered as relevant geometry for secondary stress analysis in this section. The nine-node isoperimetric two-dimension plane stress elements were used with general-purpose package SAP 2000 for the FE mesh as shown in Figure 4 . To represent the effect of no clamping forces in the rivets, the actual air gap restraint conditions were applied to represent the unilateral contact between rivet and plate. The individual deformations of rivets due to loading were not captured in this model. The uniform 


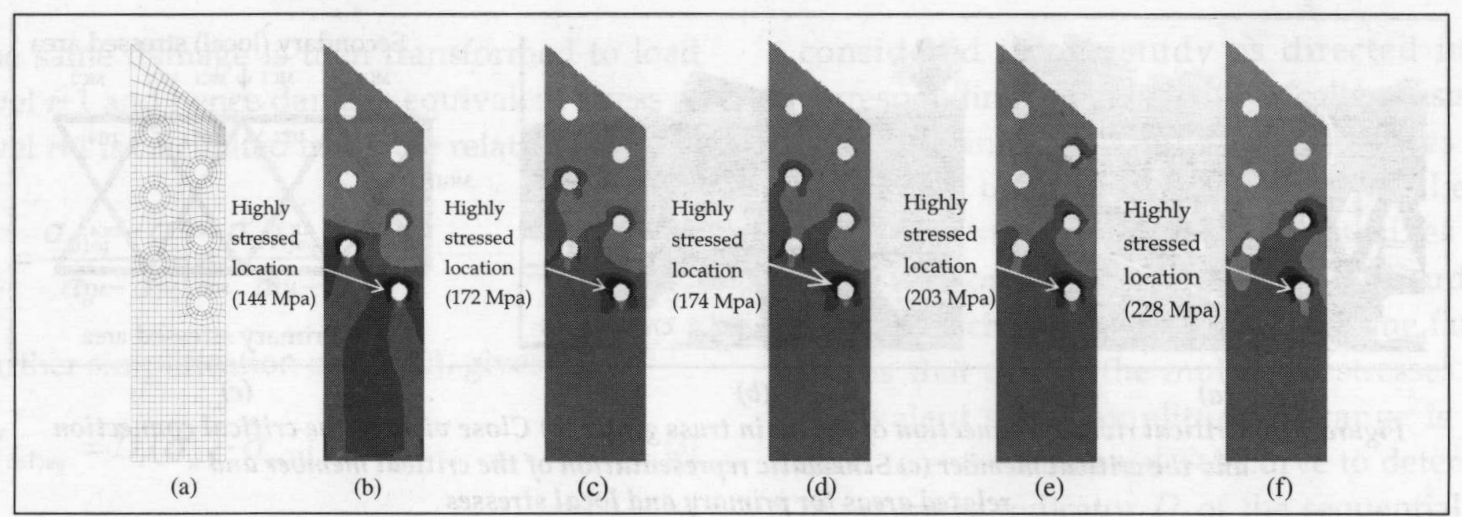

Figure 4: (a) Fine FE mesh (b) Maximum von Mises stress contour when all six rivets are active (c) Maximum von Mises stress contour when five rivets are active (d) Maximum von Mises stress contour when four rivets are active (e) Maximum von Mises stress contour when three rivets are active ( $f$ ) Maximum von Mises stress contour when two rivets are active

tensile stress history, which has been obtained from validated analytical model in detailed investigation of the bridge [14], was used as the external load of this analysis. The obtained maximum stress contours are shown in Figure 4 for considered features of riveted connection and it shows that maximum stressed locations of all the cases are subjected to elastic state of stress. Therefore, it can be said that this connection is operating in elastic state of stress with proportional loading conditions, even though this bridge is subjected to various type of rail traffic, distinct in weight and shape.

Since the proposed fatigue life estimation technique (section 2) describes the stress field at critical locations in terms of equivalent von Mises stress, the von Mises stress histories at critical location due to daily passage of trains were obtained. Sample results due to heaviest rail traffic are shown in Figure 5. The von Mises stress histories are converted in to stress ranges as mentioned in subsection 2.2 using the reservoir counting method [5].

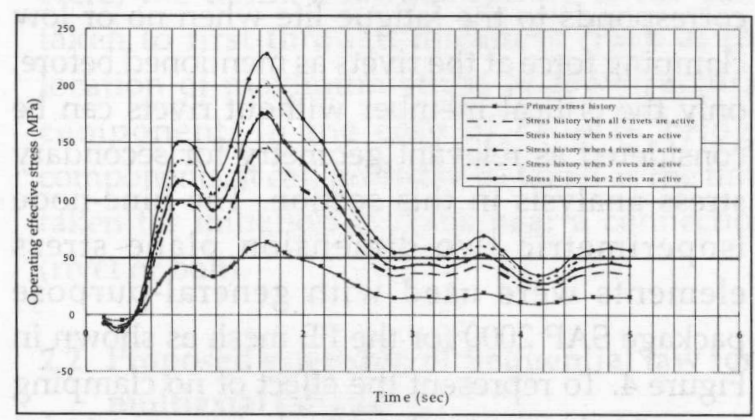

Figure 5: The von Misses stress histories at critical locations due to heaviest rail traffic for each considered features of riveted connection

\subsection{Determination of fatigue curve}

The suitable fatigue curve for this evaluation, the mean $S-N$ curve, which can be assumed to represent the case of having a low or no clamping force in the rivets, was obtained from the UK railway assessment code for Wrought iron material [13]. Further, it describes only the fatigue behavior of wrought iron material and the obtained fatigue lives from this curve show $50 \%$ probability of failure. The chosen fatigue curve only describes stress ranges, which correspond to more than ten thousands of failure cycles (usually called as partially known Wöhler curve). In the case of sequential law it is essential to know the Wöhler curve for the full range of the number of cycles. Therefore the chosen partially known Wöhler curve, which is mentioned under the UK railway assessment code [13], was transferred to fully known Wöhler curve by using Kohout and Vechet Wöhler curve modeling technique [15]. The obtained function and the geometrical shape of the new fatigue curve are illustrated in Figure 6.

\subsection{Fatigue life estimation}

A new damage indicator based sequential law in multiaxial fatigue (section 2) was utilized in this section to obtain a more realistic service life for the riveted connection. The calculated lives are shown in Table 1 and the defined fatigue life in this section describes the time duration from the date when considered feature of riveted connections appeared, to the date of fatigue failure. Further it was considered that the sequence and density of rail passage was similar to the present period of operation. 


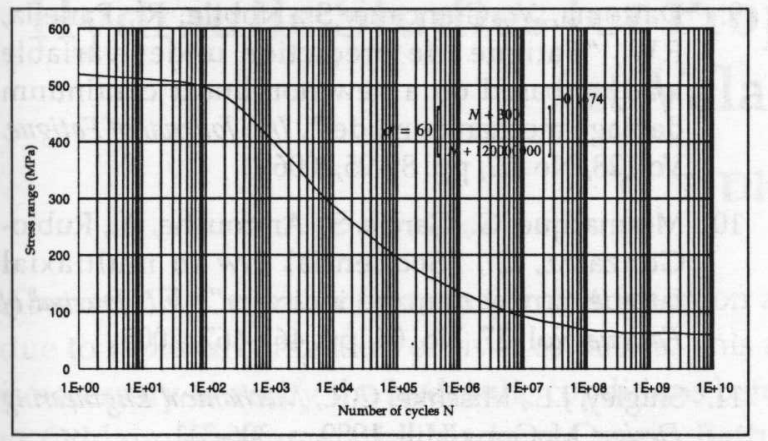

Figure 6: The mean Wöhler curve for wrought iron material (transferred to full range using the Kohout and Vechet curve modelling technique)

\section{Comparisons and Discussions}

The proposed method-based estimated fatigue lives were compared with two other previous approaches. The descriptions of the previous approaches are detailed below.

- Previous approach 1: This is based on combination of secondary stress histories, equivalent effective stress with Miner's rule [4] and railway code provided mean fatigue curve. Only a few applications has been found in history.

- Previous approach 2: This is based on combination of primary stress histories, Miner's rule [4] and modified code given fatigue curve. The above code provided mean fatigue curves are subjected to appropriate stress concentration factors (SCFs) to obtain hence the modified fatigue curves. The SCFs are determined from the ratio of the maximum principle stress (the proportion of stress opening the crack) to maximum primary stress of the critical member.

The fatigue lives, which are obtained from these two methods, are compared with the estimations made with the proposed method (see Table 1.). The defined fatigue life describes the time duration from the date when considered feature of the riveted connections appeared, to the date of fatigue failure as in previous sections. It is important to note that all these lives have been obtained by assuming all the rivets of connection have no or low clamping force.

The comparisons (Table 1) reveal that proposed method based-results deviate from both previous approaches and it shows less remaining fatigue lives than other predictions. Further, it can be seen that the lives of previous method 2 deviate significantly from predictions of the other two methods. Although these types of deviations are particular to this bridge, it can be generally said that loading sequence effect critically influence the estimation of remaining fatigue life. Additionally, it can be highlighted that the modified fatigue curves with appropriate SCFs are not able to capture the real fatigue damage due to secondary stresses precisely.

Further, the obtained fatigue lives of all the approaches (Table 1 ) reveal that the active number of rivets, which are able to transfer the load, also changes the fatigue life significantly. Generally, activeness of rivets may change with the time in service condition of a bridge and cause unexpected stress concentration near the riveted area. Therefore, when inspection and maintenance of the bridge is carried out, it is very important to investigate accurately the condition of connections wherever possible to detect unexpected stress concentration, especially in old bridges.

\section{Conclusions}

Application of the recently developed new damage indicator based sequential law was discussed in view of fatigue life assessment of riveted connection. Handling of sequential law in malti-axial fatigue of a railway bridge connection were described. The fatigue damages due to the secondary stresses of a riveted

Table 1: Fatigue life variation when active numbers of rivets are gradually decreasing while there is no effect of clamping force

\begin{tabular}{|l|c|c|c|}
\hline Considered features of the riveted connection & \multicolumn{3}{|c|}{ Remaining Fatigue life from today (months) } \\
\cline { 2 - 4 } & Proposed method & Previous method 1 & Previous method 2 \\
\hline All six rivets are active & 245 & 272 & 326 \\
Five rivets are active & 63 & 70 & 109 \\
Four rivets are active & 59 & 65 & 105 \\
Three rivets are active & 19.7 & 22.5 & 45.5 \\
Two rivets are active & 6.5 & 7.4 & 22.6 \\
\hline
\end{tabular}


connection were considered and the corresponding procedures were indicated. Finally the study leads to highlight the major conclusions as follows.

The observation and the phenomenological validity of the new damage indicator-based sequential law tend to conclude that the application of the sequential law-based proposed approach gives much more realistic remaining fatigue life of riveted bridge connections where the detailed stress histories are known. The obtained function and the geometrical shape of this fully known design S$\mathrm{N}$ curve can be employed to assess the fatigue damages of other wrought iron riveted bridges.

Since this investigation has not captured the effect of various type of micro structural changes and the effect of nanoscopic damage variables of particular material at highly stressed locations, comparisons of above approach with microsopic level fatigue theories are being worked out.

\section{References}

1. Imam, B., Righiniotis, T.D., Chryssanthopoulos, M.K., "Fatigue assessment of riveted railway bridge", Int. Journal of Steel Structures, Vol.5, No. 05, pp. 485-494, 2005.

2. Köröndi, L., Szittner, A., Kálló, M., Krisróf, L., "Determination of fatigue safety and remaining fatigue life on a riveted railway bridge by measurement", Journal of Construct. Steel Res, Vol. 46, No.1-3, paper number 327, 1998.

3. Constantine, C.S., Ioannis, G.R., John, Ch.E., "Condition assessment and retrofit of historic steel-truss railway bridges", Journal of Construct. Steel Res, Vol. 60, No.08, pp. 1213-1225, 2004.

4. Miner, M.A., "Cumulative damage in fatigue", Journal of Applied Mechanics, Vol.12, pp. 159-164, 1945.

5. BS 5400, "Steel concrete and composite bridges. Part 10: Code of practice for fatigue", British Standard Institute, 1980.

6. Fisher, J.W., Yen B.T., Wang D., "NCHRP Report No 302: Fatigue and fracture evaluation for rating riveted bridges", Transportation Research Board, National Research Council, Washington, DC, 1984.

7. Akesson, B., Fatigue life of riveted railway bridges, PhD thesis, Chalmers University of Technology, Göterborg, 1994.

8. Suresh, S., Fatigue of materials, 2nd ed., UK, Cambridge University Press, 1998.
9. Dattoma, V., Giancane, S., Nobile, R., Panella, F.W., "Fatigue life prediction under variable loading based on a new non-linear continuum damage mechanics model", Int. Journal of Fatigue, Vol. 28, No. 02, pp. 89-95,2006.

10. Mesmacque, G., Garcia. S., Amrouche, A., RubioGonzalez, C., "Sequential law in multiaxial fatigue, a new damage indicator", Int. Journal of Fatigue, Vol. 27, No. 04, pp. 461-467, 2005.

11. Shigley, J.E., Mischke, C.R., Mechanical Engineering Design, McGraw-Hill, 1989, p. 306-311.

12. Chamat, A., Abbadi, M., Gilgert, J, Cocheteux, F., Azari, Z., "A new non-local criterion in highcycle multiaxial fatigue for non-proportional loadings", Int. Journal of Fatigue, Vol. 29, No. 08, pp. 1465-1474, 2007.

13. Network Rail, RT/CE/C/025, "Rail track line code of practice: The structural assessment of under-bridges", Rail track, 2001.

14. Ranaweera, M.P.R., Aberuwan, H., Mauroof, A.L.M., Herath, K.R.B., Dissanayake, P.B.R., Siriwardane, S.A.S.C., Adasooriya, A.M.N.D., Structural appraisal of railway bridge at colombo over kelani river, Engineering Design Center, University of Peradeniya, Sri Lanka, 2002.

15. Kohout, J., Vechet S., "A new function for fatigue curves characterization and it's multiple merits", Int. Journal of Fatigue Vol. 23, No. 02, pp. 175-183, 2001. 